\title{
Phase function of a spherical particle when scattering an inhomogeneous electromagnetic plane wave
}

\section{Frisvad, Jeppe Revall}

Published in:

Journal of the Optical Society of America A

Link to article, DOI:

10.1364/JOSAA.35.000669

Publication date:

2018

Document Version

Peer reviewed version

Link back to DTU Orbit

Citation (APA):

Frisvad, J. R. (2018). Phase function of a spherical particle when scattering an inhomogeneous electromagnetic plane wave. Journal of the Optical Society of America A, 35(4), 669-680.

https://doi.org/10.1364/JOSAA.35.000669

\section{General rights}

Copyright and moral rights for the publications made accessible in the public portal are retained by the authors and/or other copyright owners and it is a condition of accessing publications that users recognise and abide by the legal requirements associated with these rights.

- Users may download and print one copy of any publication from the public portal for the purpose of private study or research.

- You may not further distribute the material or use it for any profit-making activity or commercial gain

- You may freely distribute the URL identifying the publication in the public portal

If you believe that this document breaches copyright please contact us providing details, and we will remove access to the work immediately and investigate your claim. 


\title{
Phase function of a spherical particle when scattering an inhomogeneous electromagnetic plane wave
}

\author{
Jeppe Revall Frisvad ${ }^{1, *}$ \\ ${ }^{1}$ Department of Applied Mathematics and Computer Science, Technical University of Denmark, Richard Petersens Plads, Building 321, DK-2800 Kongens \\ Lyngby, Denmark \\ *Corresponding author: jerf@dtu.dk
}

\begin{abstract}
In absorbing media, electromagnetic plane waves are most often inhomogeneous. Existing solutions for the scattering of an inhomogeneous plane wave by a spherical particle provide no explicit expressions for the scattering components. In addition, current analytical solutions require evaluation of the complex hypergeometric function ${ }_{2} F_{1}$ for every term of a series expansion. In this work, I develop a simpler solution based on associated Legendre functions with argument zero. It is similar to the solution for homogeneous plane waves but with new explicit expressions for the angular dependency of the far-field scattering components, that is, the phase function. I include recurrence formulae for practical evaluation and provide numerical examples to evaluate how well the new expressions match previous work in some limiting cases. The predicted difference in the scattering phase function due to inhomogeneity is not negligible for light entering an absorbing medium at an oblique angle. The presented theory could thus be useful for predicting scattering behavior in dye based random lasing and in solar cell absorption enhancement. () 2018 Optical Society of America. One print or electronic copy may be made for personal use only. Systematic reproduction and distribution, duplication of any material in this paper for a fee or for commercial purposes, or modifications of the content of this paper are prohibited.
\end{abstract}

OCIS codes: (290.4020) Mie theory; (290.5825) Scattering theory; (290.5850) Scattering, particles.

This is the author's version of the work. The definitive version is available at https://doi.org/10.1364/JOSAA.35.000669

\section{INTRODUCTION}

Scattering of a light wave by a perfect sphere has been considered for more than a century. The formulae were originally derived by Lorenz [1], who considered light in a nonabsorbing medium scattered by a nonabsorbing sphere. He used the ratio $N=n_{\text {med }}^{\prime} / n_{p}^{\prime}$ of the real refractive index of the host medium to that of the spherical particle. Mie [2] derived the same formulae over again using Maxwell's equations. Mie was considering colloidal suspensions of metallic particles. Since metals have a very significant imaginary part in their index of refraction, he generalized the original formulae using a complex index of refraction $n_{p}=n_{p}^{\prime}+i n_{p}^{\prime \prime}$ for the spheres.

The theory of Lorenz is useful for modeling atmospheric phenomena such as skylight, clouds, mist, haze, and rainbows. Beyond this, the types of matter consisting of nonabsorbing particles in a nonabsorbing host medium seems rather few. Mie's extension of the theory was thus a considerable improvement in terms of materials that we are able to model. The theory of scattering by spherical particles is today called the Lorenz-Mie theory (or Mie theory). Kerker [3] gives an excellent historical review of the different contributions to the theory in its initial development (and of how little the different contributors knew of each other's work).
Even with the extension of Mie, the class of materials that we can model as possibly absorbing spheres in a nonabsorbing host is somewhat limited. Many particles are approximately spheres, but oftentimes they are embedded in an absorbing host. The blue color of the seas is caused by the weak absorption of water. Hence, even water is absorbing if we consider a large enough volume. Examples of absorbing spheres in a nonabsorbing host are materials such as paints and plastics, suspensions of metallic particles in a nonabsorbing solvent, colored glass, and the like. It is useful to include the more general case where the refractive index of the host medium is also allowed to be complex. This extension of the Lorenz-Mie theory was seemingly first considered by Mundy et al. [4]. These authors used a complex index of refraction for the host medium $n_{\text {med }}=n_{\text {med }}^{\prime}+i n_{\text {med }}^{\prime \prime}$. However, they retained the assumption of a homogeneous wave and therefore ended up with angular dependency of the scattering equivalent to that of Lorenz and Mie.

After the paper by Mundy et al. [4] there has been much discussion on scattering by particles in an absorbing host [5-15]. The discussion is concerned with the problem that we cannot really consider the scattering cross section to be an independent property of the material because there is an exponential attenuation term in the direction towards the observer. It means 

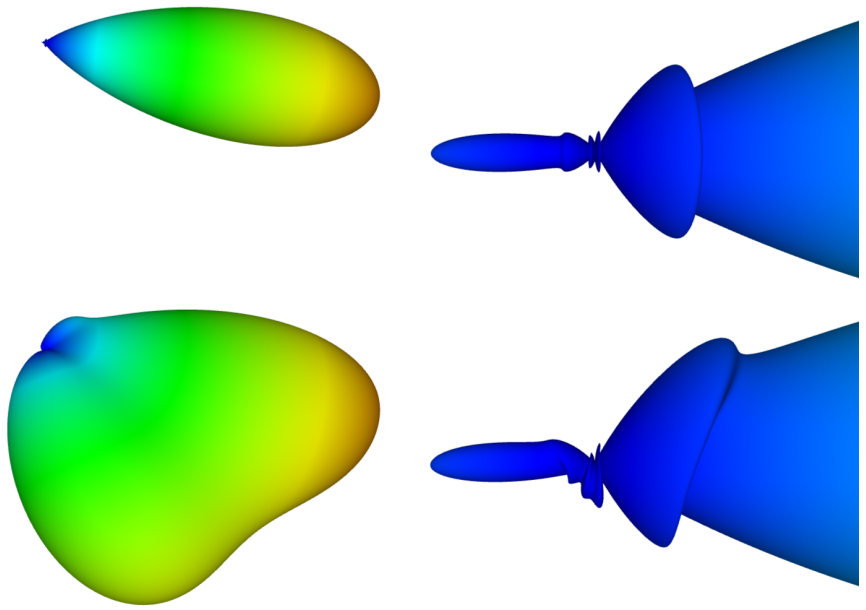

Fig. 1. The top row visualizes the Lorenz-Mie phase function for a homogeneous incident wave. The bottom row visualizes the same phase function, but taking significant inhomogeneity of the incident wave into account. Each column uses a different wavelength, type of particle, and host medium. To the left, we have the first case of Sec. 6, but with an absorbing host and a more inhomogeneous wave. To the right, we have one of the polarization components from the second case of Sec. 6 .

that we cannot really tell how much light was scattered by the particle if we look at the light that reaches a far away observer. This problem is still unsolved, and I leave it as is. I work with a different problem, namely that the authors of this previous work all assume the incident wave to be a homogeneous plane wave. Some of them [8] explicitly mention that the light should be incident on the host medium along the normal direction to ensure that this assumption holds true.

The Lorenz-Mie formulae were derived for homogeneous waves of light, but waves are only very rarely homogeneous when propagating in an absorbing medium [16]. Light refracting into an absorbing material is only homogeneous at normal incidence [17]. Belokopytov and Vasil'ev [18] have found a solution for the scattering of plane inhomogeneous waves by an absorbing particle in a nonabsorbing host. This is the special case of an evanescent wave produced by total internal reflection in a nonabsorbing medium [17-19]. As opposed to this, the goal is here to find a solution for the scattering of an absorbing sphere in an absorbing host. To do it properly, we need to take inhomogeneous waves in an absorbing medium into account.

Numerical techniques, such as the finite element method [20], are commonly used for computing the scattering of an inhomogeneous plane wave by a spherical particle in an absorbing medium. Recently, Frezza and Mangini [21] presented a solution based on vector spherical harmonics expansion of the fully general expressions for inhomogeneous plane waves. Every term of such an expansion, however, requires evaluation of the complex hypergeometrical function ${ }_{2} F_{1}$ [22], which involves an infinite sum of ratios of gamma functions. The function ${ }_{2} F_{1}$ is considered notoriously difficult to evaluate [23, 24]. Nevertheless, this previous work enables us to evaluate the scattered electromagnetic field at different positions in an absorbing medium. For applications like radiative transfer, however, it is preferable to have a computationally simpler solution and an explicit expression for the scattering phase function.

No previous work that I am aware of provides explicit for- mulae for the far-field scattering components when a spherical particle scatters an inhomogeneous wave of light. I present such formulae and provide practical recurrence relations for their evaluation. The terms of the expansion in my solution involve only sums with few terms. This makes the solution sufficiently simple to enable interactive inspection of the shape of the phase function as input parameters vary. Examples are provided in Fig. 1 and the reader is encourages to interactively explore phase function shapes. ${ }^{1}$ My solution seems a useful addition to the theory for reflection and refraction of inhomogeneous plane waves by an interface between two absorbing media [25-27]. Perhaps it can also be combined with generalized Lorenz-Mie theory [28], which is concerned with solutions for arbitrary positions of the scattering sphere in an incident beam of arbitrary shape.

The inhomogeneity of light refracting into a thin film was originally investigated to measure the optical properties of highly absorbing materials such as metals [16, 17, 25, 26]. Scattering of an inhomogeneous wave by a particle in a nonabsorbing medium [18] is useful in total internal reflection microscopy [19], as this is based on the scattering by particles of an evanescent wave. Scattering by particles in absorbing media has especially received attention in random lasing [29-32] and solar cell absorption enhancement [33-37]. In the former area, the laser gain medium is often a dye solution with embedded particles, and researchers investigate the influence of scattering on the output character of the laser. In the latter area, particles can be embedded in the active layer of a solar cell to improve the light-harvesting efficiency. The intention of this paper is to take a step toward a better understanding of the scattering by particles in absorbing media such as dyes and solar cells.

\section{PLANE WAVES OF LIGHT}

An electromagnetic plane wave is described by the real parts of the two plane wave expressions:

$$
\begin{aligned}
\mathbf{E}_{\mathcal{C}}(\mathbf{x}, t) & =\mathbf{E}_{0} e^{-i(\omega t-\mathbf{k} \cdot \mathbf{x})} \\
\mathbf{H}_{\mathcal{C}}(\mathbf{x}, t) & =\mathbf{H}_{0} e^{-i(\omega t-\mathbf{k} \cdot \mathbf{x})},
\end{aligned}
$$

where $\operatorname{Re}\left(\mathbf{E}_{\mathcal{C}}\right)$ is the electric field vector and $\operatorname{Re}\left(\mathbf{H}_{c}\right)$ is the magnetic field vector. These standard equations describe a harmonic plane wave of angular frequency $\omega$ with wave vector $\mathbf{k}$. The wave varies in time $t$ and with location in space $\mathbf{x}$. Furthermore, $\mathbf{E}_{0}$ and $\mathbf{H}_{0}$ are complex vector amplitudes and $i=\sqrt{-1}$.

Assuming isotropic materials and no charges moving freely through empty space, Maxwell's equations [38] simplify to the time-free form for plane waves [39]:

$$
\begin{aligned}
\mathbf{k} \times \mathbf{H}_{0} & =-\omega(\varepsilon+i \sigma / \omega) \mathbf{E}_{0} \\
\mathbf{k} \times \mathbf{E}_{0} & =\omega \mu \mathbf{H}_{0} \\
\mathbf{k} \cdot \mathbf{E}_{0} & =0 \\
\mathbf{k} \cdot \mathbf{H}_{0} & =0,
\end{aligned}
$$

where $\varepsilon$ is the permittivity, $\mu$ is the permeability, and $\sigma$ is the conductivity. Collectively, these three parameters are called the isotropic material properties. The time-free Maxwell equations reveal that the plane waves are required to satisfy the following conditions:

$$
\begin{gathered}
\mathbf{k} \cdot \mathbf{E}_{0}=\mathbf{k} \cdot \mathbf{H}_{0}=\mathbf{E}_{0} \cdot \mathbf{H}_{0}=0 \\
\mathbf{k} \cdot \mathbf{k}=\omega^{2} \mu(\varepsilon+i \sigma / \omega)
\end{gathered}
$$

${ }^{1}$ http://people.compute.dtu.dk/jerf/code/phase/ [This demonstrator requires a computer with a decent graphics processing unit (GPU).] 
where all the vectors may be complex and $\varepsilon_{\mathcal{C}}=\varepsilon+i \sigma / \omega$ is sometimes called the complex permittivity (or the complex dielectric constant). The latter equation is particularly interesting as it describes the relation between material and wave propagation.

Let us take a look at the real and imaginary parts of the wave vector $\mathbf{k}$. We write

$$
\mathbf{k}=\mathbf{k}^{\prime}+i \mathbf{k}^{\prime \prime}=k^{\prime} \vec{s}^{\prime}+i k^{\prime \prime} \vec{s}^{\prime \prime},
$$

where $k^{\prime}=\left|\mathbf{k}^{\prime}\right|$ and $k^{\prime \prime}=\left|\mathbf{k}^{\prime \prime}\right|$ such that $\vec{s}^{\prime}$ and $\vec{s}^{\prime \prime}$ are unit vectors in the directions of the real and imaginary parts of the wave vector, respectively. If the real part of the wave vector $\mathbf{k}^{\prime}$ is parallel to the imaginary part $\mathbf{k}^{\prime \prime}$, the wave is said to be homogeneous. Otherwise, it is inhomogeneous. Of course, $\mathbf{k}^{\prime \prime}=\mathbf{0}$ is parallel to any vector, why a wave is homogeneous if $\mathbf{k}$ is real-valued. If $\mathbf{k}$ is complex, the exponential term of the plane wave expressions (1-2) is as follows

$$
e^{i \mathbf{k} \cdot \mathbf{x}}=e^{i \mathbf{k}^{\prime} \cdot \mathbf{x}} e^{-\mathbf{k}^{\prime \prime} \cdot \mathbf{x}}
$$

Here, we may observe that $\mathbf{k}^{\prime}$ is the vector normal to the surface of constant phase and $\mathbf{k}^{\prime \prime}$ is normal to the surface of constant amplitude. The phase velocity is then $v=\omega / k^{\prime}$ and the amplitude is damped (or decays) in the direction $\vec{s}^{\prime \prime}$ at the rate $k^{\prime \prime}$. Thus, we have an inhomogeneous wave whenever the damping of the wave is not aligned with its direction of propagation.

The rule for propagation of plane waves in an isotropic medium (8) indicates the convenience of a phenomenological quantity defined by

$$
n=n^{\prime}+i n^{\prime \prime}=c \sqrt{\mu(\varepsilon+i \sigma / \omega)} .
$$

This is the (complex) index of refraction, or refractive index. Multiplication by $c$, the speed of light in vacuo, ensures that $n$ is a unitless quantity. The index of refraction nicely collects the material properties and is a quantity measured as one of the key optical properties of materials.

The energy of the field is given by the magnitude of the Poynting vector S [40]. For isotropic materials, we have

$$
|\mathbf{S}|=\varepsilon_{0} c^{2} \mu\left|\operatorname{Re}\left(\mathbf{E}_{c}\right) \times \operatorname{Re}\left(\mathbf{H}_{c}\right)\right|
$$

where $\varepsilon_{0}$ is the electric constant (also called the vacuum permittivity). The factor $\varepsilon_{0} c^{2} \mu$ is the relative permeability, which is one if the material is not magnetic. Inserting the plane wave expressions (1-2), we get

$$
\begin{aligned}
|\mathbf{S}|= & \varepsilon_{0} c^{2} \mu e^{-2 k^{\prime \prime} \vec{s}^{\prime \prime} \cdot \mathbf{x}} \\
& \times\left|\operatorname{Re}\left(\mathbf{E}_{0} e^{-i\left(\omega t-\mathbf{k}^{\prime} \cdot \mathbf{x}\right)}\right) \times \operatorname{Re}\left(\mathbf{H}_{0} e^{-i\left(\omega t-\mathbf{k}^{\prime} \cdot \mathbf{x}\right)}\right)\right| .
\end{aligned}
$$

Since the exponential terms which involve $\omega t$ and $\mathbf{k}^{\prime} \cdot \mathbf{x}$ are only oscillations, it follows that $2 k^{\prime \prime}$ is the exponential attenuation of the energy flux as the wave propagates through the material. This attenuation is called the absorption coefficient and it is often denoted by the symbol $\mu_{a}$ or $\sigma_{a}$ (these should not be confused with the permeability $\mu$ or the conductivity $\sigma$ ).

The direction of the energy flow in the electromagnetic field is generally considered to be the time average of the Poynting vector over a period of oscillation $T=2 \pi / \omega$. This is [39]

$$
\mathbf{S}_{\mathrm{avg}}=\frac{\varepsilon_{0} c^{2} \mu}{2} \operatorname{Re}\left(\mathbf{E}_{c} \times \mathbf{H}_{c}^{*}\right),
$$

where the asterisk * denotes the complex conjugate.

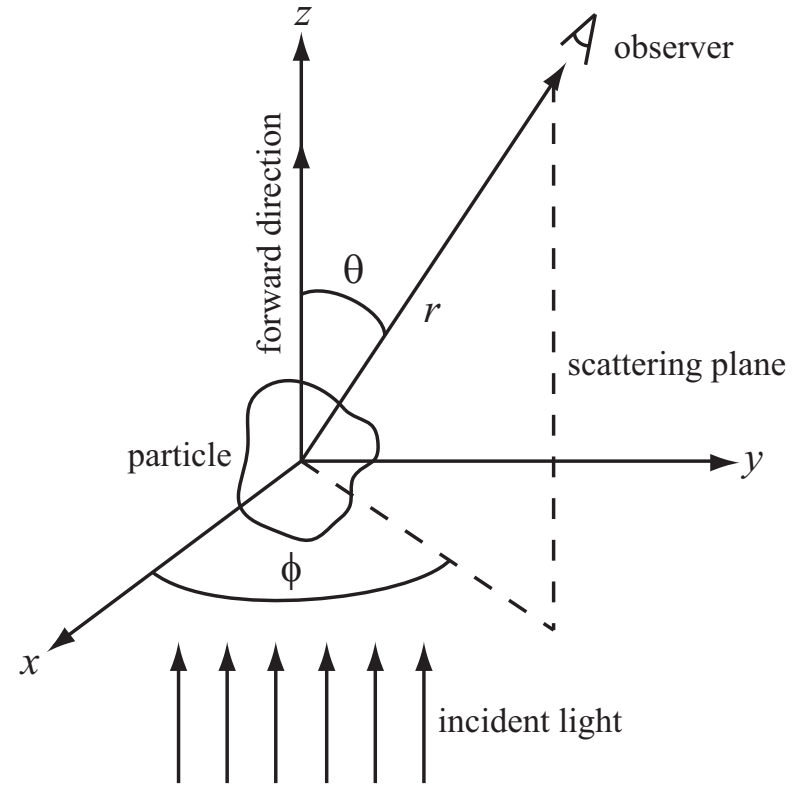

Fig. 2. Scattering by a particle.

\section{SCATTERING BY A PARTICLE}

Consider a particle in an electromagnetic field. To describe the effect, we split the field in two contributions: an incident field $\left(\mathbf{E}_{i}, \mathbf{H}_{i}\right)$ and a scattered field $\left(\mathbf{E}_{s}, \mathbf{H}_{s}\right)$. The sum of the two contributions makes up the total electromagnetic field:

$$
\mathbf{E}_{c}=\mathbf{E}_{i}+\mathbf{E}_{s}, \quad \mathbf{H}_{c}=\mathbf{H}_{i}+\mathbf{H}_{s} .
$$

Inserting in Eq. (10) to find the total time-averaged Poynting vector, we get [39]

$$
\mathbf{S}_{\mathrm{avg}}=\mathbf{S}_{i}+\mathbf{S}_{\mathrm{s}}+\mathbf{S}_{\mathrm{ext}},
$$

where $\mathbf{S}_{i}$ and $\mathbf{S}_{s}$ describe the incident and scattered fields, each with an equation as Eq. (10), but with $i$ or $s$ as subscript instead of $c$. The term $\mathbf{S}_{\text {ext }}$ describes the interaction of the two fields.

To get an idea about the directions of the different vectors, we introduce a coordinate system; see Fig. 2. The direction of the incident light (that is, the direction of $\mathbf{S}_{i}$ ) defines the axis of incidence. Let us orient our coordinate system so that it has origin inside the particle and $z$-axis along the axis of incidence. The $z$-axis is then the forward direction. Together the forward direction and the direction in which we consider the scattered light define the scattering plane. If the two directions are parallel, we may choose any plane containing the axis of incidence. In this setting, a spherical coordinate system $(r, \theta, \phi)$ is oriented such that $\theta$ is the angle between the forward direction and the scattered direction, while $\phi$ is the angle between the $x$-axis and the scattering plane. This description of the setup follows that of Bohren and Huffman [39] (Sec. 3.2).

At a large distance from the particle (in the far field) the scattered field may be expressed as a spherical wave $[39,41]$ :

$$
\mathbf{E}_{s}(\mathbf{x})=i \frac{e^{i k r}}{k r} \mathbf{Z}(\vec{s}), \quad \mathbf{H}_{s}(\mathbf{x})=\frac{k}{\omega \mu} i \frac{e^{i k r}}{k r} \vec{s} \times \mathbf{Z}(\vec{s}),
$$

where $\mathbf{x}=r \vec{s}$ such that $r$ is the distance to the observer and $\vec{s}$ is the direction toward the observer, while $\omega$ is the angular frequency, $k$ is the (complex) wave number, and $\mu$ is the permeability of the medium surrounding the particle. As a consequence 
of Eqs. (8-9) and complex number algebra, we have

$$
k=k_{0} n_{\text {med }}=\sqrt{\mathbf{k} \cdot \mathbf{k}} \neq k^{\prime}+i k^{\prime \prime},
$$

where $k_{0}=\omega / c$ is the wave number in vacuo. With $\lambda$ as the wavelength in vacuo, we also have $k_{0}=2 \pi / \lambda$. The vector $\mathbf{Z}$ denotes the strength of the light scattered in the direction $\vec{s}$. There must be a relation between the incident electric field $\mathbf{E}_{i}$ and the scattered field hidden in the directionally dependent scattering vector function $\mathbf{Z}(\vec{s})$. Let us formulate this relationship in the following paragraphs.

We consider two polarizations: one with the electric vector perpendicular to the scattering plane $(\perp, \mathrm{TE})$ and one with the electric vector parallel to the scattering plane $(\|, T M)$. Suppose we (in the case of the electric vector) denote the magnitudes of these two components by $E_{\perp i}$ and $E_{\| i}$ for the incident field and by $E_{\perp S}$ and $E_{\| S}$ for the scattered field. Then we introduce a scattering matrix $\mathbf{S}(\theta, \phi)$, which in general depends on both the angle with the forward direction $\theta$ and the location of the scattering plane $\phi$. Putting all this notation together, we get a formula describing the relationship between the magnitudes of the scattered components and those of the incident components [39, 42]:

$$
\left(\begin{array}{c}
E_{\| s} \\
E_{\perp s}
\end{array}\right)=i \frac{e^{i k r}}{k r}\left(\begin{array}{cc}
S_{2} & S_{3} \\
S_{4} & S_{1}
\end{array}\right)\left(\begin{array}{c}
E_{\| i} \\
E_{\perp i}
\end{array}\right) e^{-i \mathbf{k} \cdot \mathbf{x}},
$$

where the four-component matrix is the scattering matrix $\mathbf{S}(\theta, \phi)$ and $\mathbf{k}$ is the wave vector of the incident plane wave.

The scattering matrix gives an expression for the scattering vector function $\mathbf{Z}$ :

$$
\mathbf{Z}=\left(\begin{array}{cc}
\vec{e}_{\|} & \vec{e}_{\perp}
\end{array}\right)\left(\begin{array}{cc}
S_{2} & S_{3} \\
S_{4} & S_{1}
\end{array}\right)\left(\begin{array}{c}
E_{\| i} \\
E_{\perp i}
\end{array}\right) e^{-i \mathbf{k} \cdot \mathbf{x}},
$$

where $\vec{e}_{\|}$and $\vec{e}_{\perp}$ are unit vectors parallel and perpendicular to the scattering plane, respectively. They are chosen such that $\vec{e}_{\|} \times \vec{e}_{\perp}=\vec{s}$. Now that we have an expression connecting $\mathbf{Z}$ to the incident field, let us see how $\mathbf{Z}$ relates to the scattered energy. By insertion of the spherical wave equations (11) in the expression for the scattering Poynting vector (Eq. 10 with subscript $s$ instead of $c$ ), we get

$$
\mathbf{S}_{s}=\frac{\varepsilon_{0} c n_{\text {med }}^{\prime}}{2} \frac{e^{-2 k_{0} n_{\text {med }}^{\prime \prime} r}}{|k|^{2} r^{2}} \operatorname{Re}\left(\mathbf{Z}(\vec{s}) \times(\vec{s} \times \mathbf{Z}(\vec{s}))^{*}\right) .
$$

For comparison, the Poynting vector of the incident light field is:

$$
\mathbf{S}_{i}=\frac{\varepsilon_{0} c^{2}}{2 \omega} e^{-2 \mathbf{k}^{\prime \prime} \cdot \mathbf{x}} \operatorname{Re}\left(\mathbf{E}_{0 i} \times\left(\mathbf{k} \times \mathbf{E}_{0 i}\right)^{*}\right),
$$

Because the vectors are perpendicular, we get the following magnitudes of the scattering and incident Poynting vectors:

$$
\begin{aligned}
\left|\mathbf{S}_{s}\right| & =\frac{\varepsilon_{0} c n_{\mathrm{med}}^{\prime}}{2} \frac{e^{-2 k_{0} n_{\mathrm{med}}^{\prime \prime}}}{|k|^{2} r^{2}}|\mathbf{Z}(\vec{s})|^{2} \\
\left|\mathbf{S}_{i}\right| & =\frac{\varepsilon_{0} c n_{\mathrm{med}}^{\prime}}{2} e^{-2 \mathbf{k}^{\prime \prime} \cdot \mathbf{x}}\left|\mathbf{E}_{0 i}\right|^{2} .
\end{aligned}
$$

Let us see if we can find out something about the ratio of scattered to incident light. We denote the surface of the particle $A$. The rate of energy transfer across a closed surface arbitrarily close to $A$ is given by the integral [39]

$$
\int \mathbf{S}_{\mathrm{avg}} \cdot \vec{n} \mathrm{~d} A \text {, }
$$

where $\vec{n}$ denotes the direction of the outward surface normal. The total scattering by a particle is then an integral over the surface of the particle. A patch on the surface of the particle can be considered an area $\Delta A$ or a solid angle $\Delta \Omega$. We have

$$
\Delta \Omega=\frac{\Delta A}{r^{2}}
$$

which means that the energy scattered by a particle is given by

$$
W_{s}=\int \mathbf{S}_{s} \cdot \vec{s} \mathrm{~d} A=\int_{4 \pi} \frac{\varepsilon_{0} c n_{\text {med }}^{\prime}}{2} \frac{e^{-2 k_{0} n_{\text {med }}^{\prime \prime}}}{|k|^{2}}|\mathbf{Z}(\vec{s})|^{2} \mathrm{~d} \Omega .
$$

The conventional way of describing the ratio of scattered to incident light is to divide $W_{s}$ by the incident energy flux $\left|\mathbf{S}_{i}\right|$. To have the ratio on a simple form, we introduce a scattering vector function $\mathbf{X}$, which is like $\mathbf{Z}$, but normalized so that its expression has the weights of the two polarizations $(\|$ and $\perp$ ) rather than $E_{\| i}$ and $E_{\perp i}$ :

$$
\mathbf{X}(\vec{s})=\frac{\mathbf{Z}(\vec{s})}{\left|\mathbf{E}_{0 i}\right|} .
$$

The ratio is then

$$
C_{s}=\frac{W_{s}}{\left|\mathbf{S}_{i}\right|}=\frac{e^{-2 k_{0} n_{\text {med }}^{\prime \prime}}}{e^{-2 \mathbf{k}^{\prime \prime} \cdot \mathbf{x}}} \frac{1}{|k|^{2}} \int_{4 \pi}|\mathbf{X}(\vec{s})|^{2} \mathrm{~d} \Omega,
$$

where

$$
\mathbf{k}^{\prime \prime} \cdot \mathbf{x}=\left(k^{\prime \prime} \vec{s}^{\prime \prime}\right) \cdot(r \vec{s}) \approx k_{0} n_{\text {med }}^{\prime \prime} r \frac{\vec{s} \cdot \vec{s}^{\prime \prime}}{\vec{s}^{\prime} \cdot \vec{s}^{\prime \prime}} \approx k_{0} n_{\text {med }}^{\prime \prime} r,
$$

why we assume that the exponential term in the expression for $C_{S}$ is 1. A ratio of total scattered energy to energy incident per unit area (15) gives an area as result. This particular area $C_{S}$ is referred to as the scattering cross section of the particle. It is the area that would receive the same amount of energy as the particle scatters if we subtend it normal to the incident light.

If we normalize the directionally dependent quantity under the integral, we have the phase function of the particle:

$$
p(\theta, \phi)=\frac{|\mathbf{X}(\theta, \phi)|^{2}}{|k|^{2} C_{s}}
$$

Let us now turn to the idealized case of a spherical particle.

\section{SCATTERING BY A SPHERE}

Consider a plane wave scattered by a spherical particle embedded in a host medium. We take it that both the host medium and the particle are isotropic, homogeneous substances. Plane waves would otherwise not be a good approximation. Note that we make no assumptions about the absorption properties of the host medium and the particle, and we do not require the plane wave to be homogeneous or inhomogeneous.

We are aiming at a solution for the scattering phase function. As seen in Eq. (16), this requires the normalization factor $|k|^{2} C_{S}$ and the components of the scattering matrix $\mathbf{S}(\theta, \phi)$.

In Sec. 3 (Fig. 2), we chose our coordinate system so that light is incident along the $z$-axis. This means that the $z$-axis points along the direction of $\mathbf{S}_{i}$. For homogeneous plane waves, the direction of $\mathbf{S}_{i}$ is parallel to both the real and imaginary parts of the wave vector, but in general this is not necessarily the case. This means that the wave vector $\mathbf{k}$ has two different directions: one for the real part $\mathbf{k}^{\prime}$ and one for the imaginary part $\mathbf{k}^{\prime \prime}$.

The scattering plane is spanned by the z-axis (forward direction) and the direction of the scattered light. Since we are 


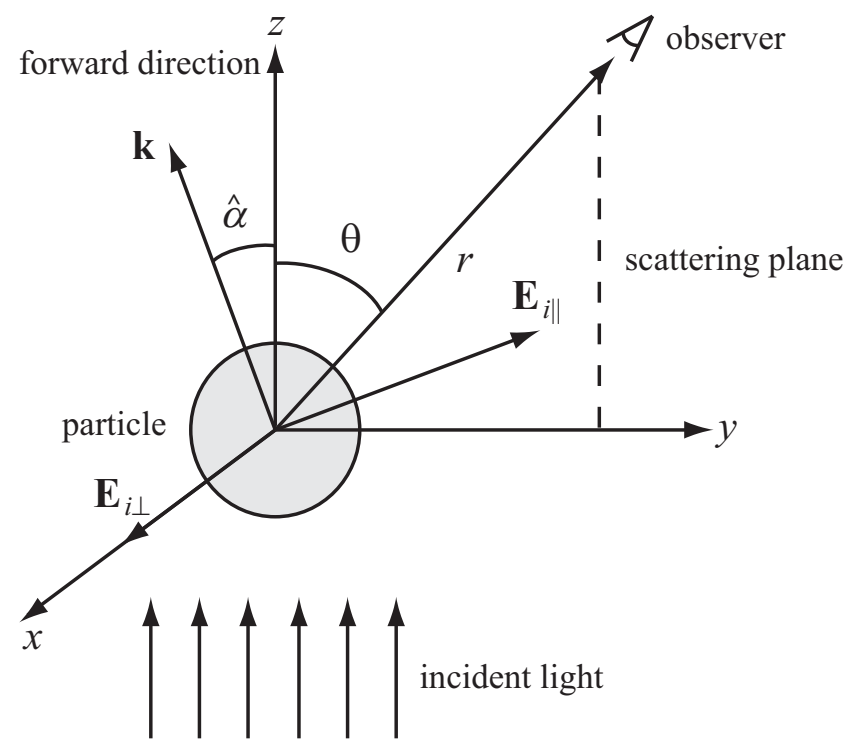

Fig. 3. Scattering by a spherical particle in an absorbing host.

working with a sphere, it is of no consequence with respect to particle geometry which way we choose to orient the $x$ and $y$ axes of the coordinate system. We are therefore always allowed to perform a change of basis and choose new directions for the $x$ and $y$ axes. Suppose we choose for any point of observation to align the $y z$-plane with the scattering plane through a change of basis. We can then find the coordinates of the vectors in the new basis through projection. In this coordinate system, where the $y z$-plane is aligned with the scattering plane, it is convenient to specify an arbitrary incident plane wave by

$$
\mathbf{E}_{i}=\mathbf{E}_{0 i} e^{-i\left(\omega t-k_{x} x\right)} e^{i\left(k_{y} y+k_{z} z\right)} .
$$

We can think of it as a wave with wave vector $\mathbf{k}=\left(0, k_{y}, k_{z}\right)$ and amplitude that varies in time and with the location along the $x$-axis. Note that this wave vector corresponds exactly to the projection of an arbitrary wave vector on the scattering plane spanned by the forward direction and the direction toward the observer. Note also that, in the end, we are only interested in evaluating this wave at the point of observation, where $x=0$ with our particular choice of basis.

Electromagnetic waves are transverse (due to Maxwell's equations). Any inhomogeneous transverse plane wave can be decomposed into a transverse electric wave (TE) and a transverse magnetic (TM) wave [26]. We now decompose our incident wave in this way. As before, we identify TE components with $\perp$-polarized components and TM components with $\|$-polarized components. The $\perp$-polarized component of the electric field vector is by definition perpendicular to the scattering plane. The $\|$-polarized component lies in the scattering plane. We have

$$
\mathbf{E}_{i}=E_{\perp i} \vec{e}_{x}+E_{\| i}\left(a \vec{e}_{y}+b \vec{e}_{z}\right),
$$

where $\vec{e}_{x}, \vec{e}_{y}$, and $\vec{e}_{z}$ are unit vectors in the directions of the coordinate axes, $E_{\perp i}$ and $E_{\| i}$ are complex magnitudes of the two polarization components of $\mathbf{E}_{i}$, and $a$ and $b$ are the direction cosines of $\mathbf{E}_{\| i}$ in the $y$ and $z$ directions, respectively. See Fig. 3. For a homogeneous wave, the direction of $\mathbf{E}_{\| i}$ would also be perpendicular to the $z$-axis, but this is not true in general.

The direction cosines $a$ and $b$ are given by

$$
a=E_{y \| i} / E_{\| i} \quad \text { and } \quad b=E_{z \| i} / E_{\| i} .
$$

Using that the $\|$-polarized component is also transverse magnetic (TM), we have

$$
H_{y \| i}=H_{z \| i}=0 \quad \text { and } \quad H_{x \| i}=H_{\| i}=\sqrt{\mathbf{H}_{\| i} \cdot \mathbf{H}_{\| i}} .
$$

Inserting this in Eq. (3) and considering the magnitude of the (complex) wave vector (8), we obtain

$$
k_{z} H_{\| i}=-\frac{k^{2}}{\omega \mu} E_{y \| i} \text { and } k_{y} H_{\| i}=\frac{k^{2}}{\omega \mu} E_{z \| i}
$$

from which the following expressions appear:

$$
a=-\frac{k_{z} \omega \mu}{k^{2}} \frac{H_{\| i}}{E_{\| i}}, \quad b=\frac{k_{y} \omega \mu}{k^{2}} \frac{H_{\| i}}{E_{\| i}} .
$$

The ratio of the amplitude magnitudes of the (complex) electric and magnetic vectors is obtained from Eqs. (4-5):

$$
\sqrt{\left(\mathbf{k} \times \mathbf{E}_{\| i}\right) \cdot\left(\mathbf{k} \times \mathbf{E}_{\| i}\right)}=k E_{\| i}=\omega \mu H_{\| i} .
$$

Insertion leads to

$$
a=-\frac{k_{z}}{k_{0} n_{\text {med }}}=-\cos \hat{\alpha}, b=\frac{k_{y}}{k_{0} n_{\text {med }}}=\sin \hat{\alpha},
$$

where $\hat{\alpha}$ is a complex angle denoting the direction of the wave vector in the scattering plane (Fig. 3). In the following, I use a Greek letter with a hat for complex angles.

The structure of the electric vector of the incident wave (17) is quite different from the case of a homogeneous wave, where only one spatial coordinate is left in the exponential. For the inhomogeneous wave the scattering is not axially symmetric around the forward direction. This is counterintuitive as the geometry of the scatterer is perfectly symmetric. The inhomogeneity of the wave is enough to break the symmetry. As a consequence, we can use only part of the results of Lorenz and Mie (and many others) for inhomogeneous waves. To find a solution, we look to the work of Belokopytov and Vasil'ev [18].

Suppose we introduce the following slightly unusual set of spherical coordinates:

$$
\begin{aligned}
& x=r \cos \beta \\
& y=r \sin \beta \sin \theta \\
& z=r \sin \beta \cos \theta,
\end{aligned}
$$

where the $x$-axis is the polar axis such that the scattering plane is given by $\sin \beta=1$ and the forward direction when also $\theta=0$. The angle $\theta$ is shown in Fig. 3. With these spherical coordinates, the expression for our incident field is

$$
\mathbf{E}_{i}=\mathbf{E}_{0 i} e^{-i\left(\omega t-k_{x} x\right)} e^{i r\left(k_{y} \sin \beta \sin \theta+k_{z} \sin \beta \cos \theta\right)} .
$$

Using the complex angle $\hat{\alpha}$, we can also write it as

$$
\begin{aligned}
\mathbf{E}_{i} & =\mathbf{E}_{0 i} e^{-i\left(\omega t-k_{x} x\right)} e^{i k r \sin \beta(\sin \hat{\alpha} \sin \theta+\cos \hat{\alpha} \cos \theta)} \\
& =\mathbf{E}_{0 i} e^{-i\left(\omega t-k_{x} x\right)} e^{i k r \sin \beta \cos (\theta-\hat{\alpha})}
\end{aligned}
$$

This is very close to the form of incident wave for which Belokopytov and Vasil'ev found a solution [18]. The generalization is in the definition of $\hat{\alpha}$ (which is purely imaginary in their treatment). For an inhomogeneous wave, $\mathbf{k}^{\prime}$ and $\mathbf{k}^{\prime \prime}$ are not parallel and a nonabsorbing medium has $\mathbf{k} \cdot \mathbf{k}=k_{0}^{2} n_{\text {med }}^{2}$ to be a real number. This can be fulfilled by having $k_{z}$ real and $k_{y}$ purely imaginary, and then the structure of our incident wave becomes equivalent to the one addressed by Belokopytov and Vasil'ev. 
Using a formula due to Bauer [43], we now expand the exponential part of the plane inhomogeneous incident wave in spherical harmonics $[1,2,18,41]$ :

$$
e^{i k r \sin \beta \cos (\theta-\hat{\alpha})}=\sum_{n=0}^{\infty} i^{n}(2 n+1) \frac{\psi_{n}(k r)}{k r} P_{n}(\sin \beta \cos (\theta-\hat{\alpha})) .
$$

The functions $\psi_{n}$ are Riccati-Bessel functions, which we will return to later. The wave number $k=k_{0} n_{\text {med }}$ is complex for an absorbing host, but that does not invalidate the expansion. In the case of homogeneous waves, the argument for the Legendre polynomials $P_{n}$ are real-valued. This is not the case for inhomogeneous waves. We would like to avoid evaluating the Legendre polynomials for complex arguments, as this would lead to the computationally difficult solutions employed in previous work [21,22]. We therefore use the addition theorem for spherical harmoics to obtain [18]:

$$
\begin{aligned}
& P_{n}(\sin \beta \cos (\theta-\hat{\alpha}))=P_{n}(\cos \beta) P_{n}(0) \\
& \quad+2 \sum_{m=1}^{n} \frac{(n-m) !}{(n+m) !} P_{n}^{m}(\cos \beta) P_{n}^{m}(0) \cos (m(\theta-\hat{\alpha})),
\end{aligned}
$$

where $P_{n}^{m}$ are the associated Legendre polynomials. Based on Abramowitz and Stegun [44], we find

$$
P_{n}^{m}(0)=(-1)^{(n+m) / 2} \frac{2^{-n}(n+m) !}{[(n-m) / 2] ![(n+m) / 2] !}
$$

for $n+m$ even and

$$
P_{n}^{m}(0)=0 \quad \text { for } n+m \text { odd. }
$$

To shorten notation it is convenient to introduce

$$
R_{n}^{m}=(-1)^{(n+m) / 2} \frac{2^{1-n-\delta_{m 0}}(n-m) !}{[(n-m) / 2] ![(n+m) / 2] !}
$$

for $n+m$ even and

$$
R_{n}^{m}=0 \quad \text { for } n+m \text { odd, }
$$

where $\delta_{m 0}$ is a Kronecker delta which is 1 when $m=0$ and 0 otherwise. Then [18]

$$
P_{n}(\sin \beta \cos (\theta-\hat{\alpha}))=\sum_{m=0}^{n} R_{n}^{m} P_{n}^{m}(\cos \beta) \cos (m(\theta-\hat{\alpha})) .
$$

What we are really interested in is the scattered wave. In particular, we would like to determine the four components of the scattering matrix $\mathbf{S}(\theta, \phi)$. It has been shown many times in the literature that all the components of the scattered field can be found using the Debye potentials [3, 41, 42, 45]. In the expansion of Belokopytov and Vasil'ev [18], the Debye potentials of the scattered wave outside the sphere are:

$$
\begin{aligned}
u & =\frac{1}{k^{2}} \sum_{n=1}^{\infty} i^{n-1} \frac{2 n+1}{n(n+1)} a_{n} \zeta_{n}(k r) U_{n}(\theta, \beta) \\
v & =\frac{1}{k k_{0} \mu} \sum_{n=1}^{\infty} i^{n-1} \frac{2 n+1}{n(n+1)} b_{n} \zeta_{n}(k r) V_{n}(\theta, \beta),
\end{aligned}
$$

where

$$
\begin{aligned}
U_{n}(\theta, \beta) & =\left(E_{\| i} W_{1 n}(\theta, \beta)+E_{\perp i} W_{2 n}(\theta, \beta)\right) e^{-i \mathbf{k} \cdot \mathbf{x}} \\
V_{n}(\theta, \beta) & =\left(E_{\| i} W_{2 n}(\theta, \beta)-E_{\perp i} W_{1 n}(\theta, \beta)\right) e^{-i \mathbf{k} \cdot \mathbf{x}} \\
W_{1 n}(\theta, \beta) & =\sum_{m=0}^{n} m R_{n}^{m} P_{n}^{m}(\cos \beta) \sin (m(\theta-\alpha)) \\
W_{2 n}(\theta, \beta) & =\sum_{m=0}^{n}(n-m) R_{n-1}^{m} P_{n}^{m}(\cos \beta) \cos (m(\theta-\alpha)) .
\end{aligned}
$$

The exponential terms in Eqs. (27-28) appear to cancel the corresponding but reciprocal exponential terms in $E_{\| i}$ and $E_{\perp i}$. The spherical function $\zeta_{n}$ is another Riccati-Bessel function and the coefficients $a_{n}$ and $b_{n}$ are the traditional Lorenz-Mie coefficients. We will return to those shortly.

Far field expressions for the magnitudes of the two polarization components of the scattered field $\left(E_{s \|}\right.$ and $\left.E_{s \perp}\right)$ lead to expressions for the components in the scattering matrix (see Eq. 12). We have $[39,42]$

$$
E_{\| s}=-E_{\theta} \quad \text { and } \quad E_{\perp s}=E_{\beta}
$$

with flipped signs because of our unusual choice of spherical coordinate system, and using the Debye potentials we also have $[3,41]$

$$
\begin{aligned}
& E_{\beta}=\frac{1}{r} \frac{\partial^{2} u}{\partial r \partial \beta}+i k_{0} \mu \frac{1}{r \sin \beta} \frac{\partial v}{\partial \theta} \\
& E_{\theta}=\frac{1}{r \sin \beta} \frac{\partial^{2} u}{\partial r \partial \theta}-i k_{0} \mu \frac{1}{r} \frac{\partial v}{\partial \beta} .
\end{aligned}
$$

Considering the relations (31) between these components and the expressions for $E_{s \|}$ and $E_{s \perp}$ from Eq. (12), we obtain equations which involve the components of the scattering matrix. In the far field, we have [3]

$$
\zeta_{n}(k r) \approx i^{-n-1} e^{i k r} \quad \text { and } \quad \frac{\partial \zeta_{n}(k r)}{\partial r} \approx i^{-n} k e^{i k r} .
$$

Inserting this far field approximation (34) in the Debye potentials (25-26) and inserting the potentials in the expressions for $E_{\beta}$ and $E_{\theta}$ (32-33), the equations with the components of the scattering matrix simplify to

$$
\begin{aligned}
& \left(S_{4} E_{\| i}+S_{1} E_{\perp i}\right) e^{-i \mathbf{k} \cdot \mathbf{x}} \\
& \quad=\sum_{n=1}^{\infty} \frac{2 n+1}{n(n+1)}\left(-a_{n} \frac{\partial U_{n}}{\partial \beta}-b_{n} \frac{1}{\sin \beta} \frac{\partial V_{n}}{\partial \theta}\right) \\
& \left(S_{2} E_{\| i}+S_{3} E_{\perp i}\right) e^{-i \mathbf{k} \cdot \mathbf{x}} \\
& \quad=\sum_{n=1}^{\infty} \frac{2 n+1}{n(n+1)}\left(a_{n} \frac{1}{\sin \beta} \frac{\partial U_{n}}{\partial \theta}-b_{n} \frac{\partial V_{n}}{\partial \beta}\right) .
\end{aligned}
$$

Using the expressions (27-30) for the $U_{n}$ and $V_{n}$ functions, we arrive at the following scattering matrix components:

$$
\begin{aligned}
& S_{1}=\sum_{n=1}^{\infty} \frac{2 n+1}{n(n+1)}\left(-a_{n} \frac{\partial W_{2 n}}{\partial \beta}+b_{n} \frac{1}{\sin \beta} \frac{\partial W_{1 n}}{\partial \theta}\right) \\
& S_{2}=\sum_{n=1}^{\infty} \frac{2 n+1}{n(n+1)}\left(a_{n} \frac{1}{\sin \beta} \frac{\partial W_{1 n}}{\partial \theta}-b_{n} \frac{\partial W_{2 n}}{\partial \beta}\right) \\
& S_{3}=\sum_{n=1}^{\infty} \frac{2 n+1}{n(n+1)}\left(a_{n} \frac{1}{\sin \beta} \frac{\partial W_{2 n}}{\partial \theta}+b_{n} \frac{\partial W_{1 n}}{\partial \beta}\right) \\
& S_{4}=\sum_{n=1}^{\infty} \frac{2 n+1}{n(n+1)}\left(-a_{n} \frac{\partial W_{1 n}}{\partial \beta}-b_{n} \frac{1}{\sin \beta} \frac{\partial W_{2 n}}{\partial \theta}\right) .
\end{aligned}
$$

Let us interpret the angles appearing in this generalized formulation of the Lorenz-Mie theory. Using a change of basis depending on the point of observation, we ensured that the $x$ axis is along the direction of the $\perp$-polarized component of the electric vector. The angle $\beta$ is the polar angle between this component and the scattering plane. Since we are only evaluating the wave at the point of observation (where $x=0$ ), this is a right angle, and thus $\beta=\pi / 2$. The azimuth angle $\theta$ is the angle in the scattering plane between the forward direction and the 
direction of the scattered light. This means that $\theta$ is the standard scattering angle. Another angle in the formulae is the complex angle $\hat{\alpha}$. It is a measure of the inhomogeneity of the incident wave, and it is given by the direction cosines in the scattering plane of the wave vector $\mathbf{k}$ of the incident light (Eq. 19).

It should be mentioned that for a homogeneous wave, we get $k_{z}=k$ and $k_{y}=0$, and then $\hat{\alpha}=0$. This simplifies the structure of the exponential term in the expression for the incident wave, and we get the components of the scattering matrix in the commonly available form [1-3, 42]:

$$
\begin{aligned}
& S_{1}(\theta)=\sum_{n=1}^{\infty} \frac{2 n+1}{n(n+1)}\left(a_{n} \pi_{n}(\cos \theta)+b_{n} \tau_{n}(\cos \theta)\right) \\
& S_{2}(\theta)=\sum_{n=1}^{\infty} \frac{2 n+1}{n(n+1)}\left(a_{n} \tau_{n}(\cos \theta)+b_{n} \pi_{n}(\cos \theta)\right),
\end{aligned}
$$

where the functions $\pi_{n}$ and $\tau_{n}$ are related to the Legendre polynomials $P_{n}$ as follows:

$$
\begin{aligned}
\pi_{n}(\cos \theta) & =\frac{P_{n}^{1}(\cos \theta)}{\sin \theta}=\frac{\mathrm{d} P_{n}(\cos \theta)}{\mathrm{d}(\cos \theta)} \\
\tau_{n}(\cos \theta) & =\frac{\mathrm{d} P_{n}^{1}(\cos \theta)}{\mathrm{d} \theta} \\
& =\cos \theta \pi_{n}(\cos \theta)-\sin ^{2} \theta \frac{\mathrm{d} \pi_{n}(\cos \theta)}{\mathrm{d}(\cos \theta)} .
\end{aligned}
$$

Their numeric evaluation can be found in standard references on Lorenz-Mie theory $[39,46]$.

It remains in this discussion of scattering by a spherical particle to give expressions for the Lorenz-Mie coefficients $a_{n}$ and $b_{n}$. In the far field, they are given by the same formulae that Lorenz [1] derived more than a century ago, only now the refractive indices are complex:

$$
\begin{aligned}
a_{n} & =\frac{n_{\mathrm{med}} \psi_{n}^{\prime}(y) \psi_{n}(x)-n_{p} \psi_{n}(y) \psi_{n}^{\prime}(x)}{n_{\mathrm{med}} \psi_{n}^{\prime}(y) \zeta_{n}(x)-n_{p} \psi_{n}(y) \zeta_{n}^{\prime}(x)} \\
b_{n} & =\frac{n_{p} \psi_{n}^{\prime}(y) \psi_{n}(x)-n_{\mathrm{med}} \psi_{n}(y) \psi_{n}^{\prime}(x)}{n_{p} \psi_{n}^{\prime}(y) \zeta_{n}(x)-n_{\mathrm{med}} \psi_{n}(y) \zeta_{n}^{\prime}(x)}
\end{aligned}
$$

where the primes ' denote derivative. The Riccati-Bessel functions $\psi_{n}(z)$ and $\zeta_{n}(z)$ are related to the spherical Bessel functions $j_{n}(z)$ and $y_{n}(z)$ as follows:

$$
\begin{aligned}
& \psi_{n}(z)=z j_{n}(z) \\
& \zeta_{n}(z)=z\left(j_{n}(z)+i y_{n}(z)\right) .
\end{aligned}
$$

One should use the complex conjugate of $\zeta_{n}$ if the time dependence for the wave had been taken to be $e^{i \omega t}$ instead of $e^{-i \omega t}$. The argument $z$ is an arbitrary complex number, the arguments $x$ and $y$ used for the Lorenz-Mie coefficients are related to particle and host media as follows:

$$
x=\frac{2 \pi r n_{\text {med }}}{\lambda} \text { and } y=\frac{2 \pi r n_{p}}{\lambda},
$$

where $\lambda$ is the wavelength in vacuo and $r$ is the radius of the spherical particle.

When computers came around, it turned out to be quite difficult to find a numerically stable way of evaluating the spherical functions $\psi_{n}$ and $\zeta_{n}$ for complex arguments. Eventually, the numerical difficulties were solved for complex $y$ [46-48]. This is sufficient for the traditional Lorenz-Mie theory with a nonabsorbing host medium. When people started considering spheres in an absorbing host, starting with Mundy et al. [4], it became necessary to find a robust way of evaluating the Lorenz-Mie coefficients for complex $x$ as well. A robust evaluation scheme has been proposed by Frisvad et al. [49].

To complete the phase function solution, we still miss the normalization factor $|k|^{2} C_{s}$ and the calculation of the complex angle $\hat{\alpha}$ by means of projection. With the Lorenz-Mie coefficients, we can find the normalization factor for the phase function using [39]

$$
|k|^{2} C_{s}=2 \pi \sum_{n=1}^{\infty}(2 n+1)\left(\left|a_{n}\right|^{2}+\left|b_{n}\right|^{2}\right) .
$$

Suppose we let $\mathbf{k}_{i}$ denote the arbitrary wave vector of the incident field before projection to the scattering plane. The projected wave vector is then given by

$$
\mathbf{k}=\mathbf{k}_{i}-\frac{\mathbf{k}_{i} \cdot\left(\mathbf{S}_{i} \times \vec{s}\right)}{\left|\mathbf{S}_{i} \times \vec{s}\right|^{2}} \mathbf{S}_{i} \times \vec{s}
$$

with $\mathbf{k}=\mathbf{k}_{i}$ if $\left|\mathbf{S}_{i} \times \vec{s}\right|=0$. After this projection, we can find the complex angle $\hat{\alpha}$ using dot products and arcus cosine for complex numbers

$$
\hat{\alpha}=\cos ^{-1} \frac{\mathbf{S}_{i} \cdot \mathbf{k}}{\left|\mathbf{S}_{i}\right| \sqrt{\mathbf{k} \cdot \mathbf{k}}} .
$$

In this setting, we can calculate $\vec{s}$ from the spherical coordinates $\theta$ and $\phi$ if we want angles as arguments for the phase function.

\section{SIMPLIFICATION}

Given that we always have $\beta=\pi / 2$, we should try to simplify the solution presented in Equations 35-38. What we are interested in is the partial derivatives of the functions $W_{1 n}$ and $W_{2 n}$ (Equations 29-30). This means that we need the derivatives

$$
\begin{aligned}
& \frac{\mathrm{d}}{\mathrm{d} \theta}(\sin (m(\theta-\hat{\alpha}))=m \cos (m(\theta-\hat{\alpha})) \\
& \frac{\mathrm{d}}{\mathrm{d} \theta}(\cos (m(\theta-\hat{\alpha}))=-m \sin (m(\theta-\hat{\alpha}))
\end{aligned}
$$

and

$$
\frac{\mathrm{d}}{\mathrm{d} \beta} P_{n}^{m}(\cos \beta)=-\sin \beta \frac{\mathrm{d} P_{n}^{m}}{\mathrm{~d} x}(x)=-\frac{\mathrm{d} P_{n}^{m}}{\mathrm{~d} x}(0),
$$

where $x=\cos \beta$. The recurrence relations for the associated Legendre polynomials provide excellent tools for evaluating the special case of zero as argument for the associated Legendre polynomials and their derivatives. Using the relations

$$
\begin{aligned}
\left(x^{2}-1\right) \frac{\mathrm{d} P_{n}^{m}}{\mathrm{~d} x}(x)= & \sqrt{1-x^{2}} P_{n}^{m+1}(x)+m x P_{n}^{m}(x) \\
\left(x^{2}-1\right) \frac{\mathrm{d} P_{n}^{m}}{\mathrm{~d} x}(x)= & -(n+m)(n-m+1) \\
& \times \sqrt{1-x^{2}} P_{n}^{m-1}(x)-m x P_{n}^{m}(x),
\end{aligned}
$$

we get the following simple relations for the special case of $\beta=\pi / 2$ :

$$
\begin{aligned}
\frac{\mathrm{d}}{\mathrm{d} \beta} P_{n}^{m}\left(\cos \frac{\pi}{2}\right) & =-\frac{\mathrm{d} P_{n}^{m}}{\mathrm{~d} x}(0)=P_{n}^{m+1}(0) \\
\frac{\mathrm{d}}{\mathrm{d} \beta} P_{n}^{n}\left(\cos \frac{\pi}{2}\right) & =-\frac{\mathrm{d} P_{n}^{n}}{\mathrm{~d} x}(0)=0 .
\end{aligned}
$$

This means that, if we have the zero argument values of the associated Legendre polynomials (Equations 21-22, Fig. 4), the zero argument derivatives with respect to the angle are given 


\begin{tabular}{|c|c|c|c|c|c|c|c|c|c|}
\hline$n \backslash m$ & -4 & -3 & -2 & -1 & 0 & 1 & 2 & 3 & 4 \\
\hline 0 & & & & & 1 & & & & \\
\hline 1 & & & & $1 / 2$ & 0 & -1 & & & \\
\hline 2 & & & $1 / 8$ & 0 & $-1 / 2$ & 0 & 3 & & \\
\hline 3 & & $1 / 48$ & 0 & $-1 / 8$ & 0 & $3 / 2$ & 0 & -15 & \\
\hline 4 & $1 / 384$ & 0 & $-1 / 48$ & 0 & $3 / 8$ & 0 & $-15 / 2$ & 0 & 105 \\
\hline
\end{tabular}

Fig. 4. The zero argument values of the associated Legendre polynomials $P_{n}^{m}(0)$ for $n=0,1,2,3,4$ and $m=-4,-3, \ldots, 3,4$.

by a right hand shift (or inserting zero if there is no right hand neighbour).

The recurrence relations also provide simple formulae for finding the values of the associated Legendre polynomials themselves. Using the relations

$$
\begin{aligned}
& P_{0}^{0}(x)=1 \\
& P_{n+1}^{n}(x)=x(2 n+1) P_{n}^{n}(x) \\
& \sqrt{1-x^{2}} P_{n}^{m+1}(x)=(n-m) x P_{n}^{m}(x)-(n+m) P_{n-1}^{m}(x) \\
& 2 m x P_{n}^{m}(x)=\sqrt{1-x^{2}} \\
& \times\left[(n+m)(m-n-1) P_{n}^{m-1}(x)-P_{n}^{m+1}(x)\right]
\end{aligned}
$$

we get the following relations for the special case of zero argument:

$$
\begin{aligned}
P_{0}^{0}(0) & =1 \\
P_{n+1}^{n}(0) & =0 \\
P_{n}^{m+1}(0) & =-(n+m) P_{n-1}^{m}(0) \\
P_{n}^{m-1}(0) & =(n+m)^{-1}(m-n-1)^{-1} P_{n}^{m+1}(0) .
\end{aligned}
$$

These four relations provide a simple forward recurrence for computing the zero argument values of all the associated Legendre polynomials. Placing the values in a triangle as illustrated in Fig. 4, the algorithm starts with the initial value (47), takes a diagonal step down and to the right to find the last element of the next row (49), inserts a zero before the last element of the row (48), fills out the remaining elements of the row (50), and goes back to the last element from where it takes a new diagonal step (49). This continues until all desired rows have been filled.

The values in Fig. 4 can also be calculated using Equations 2122. From a numerical standpoint, the recurrence algorithm (47-50) is significantly more attractive as it avoids the factorial function. To make the evaluation of $R_{n}^{m}$ equally attractive, we find from Equations 21-24 that

$$
R_{n}^{m}=2^{1-\delta_{m 0}}(-1)^{m} P_{n}^{-m}(0)
$$

These equations also inform us that when $n+m$ is an odd number, the values of $P_{n}^{m}(0)$ and $R_{n}^{m}$ are zero. Thus $R_{n-1}^{m} P_{n}^{m}(0)$ are zero, and $R_{n}^{m} \frac{\mathrm{d} P_{n}^{m}(0)}{\mathrm{d} \beta}$ are also zero due to Equation 46 . This means that the functions $W_{2 n}(\theta, \pi / 2)$ vanish unless we take the partial derivative with respect to $\beta$, whence $W_{1 n}(\theta, \pi / 2)$ would vanish instead. From this, we conclude that

$$
S_{3}=S_{4}=0 .
$$

Equation 21 also enables us to simplify the expressions for the scattering components $S_{1}$ and $S_{2}$ (35-36). In fact, we can

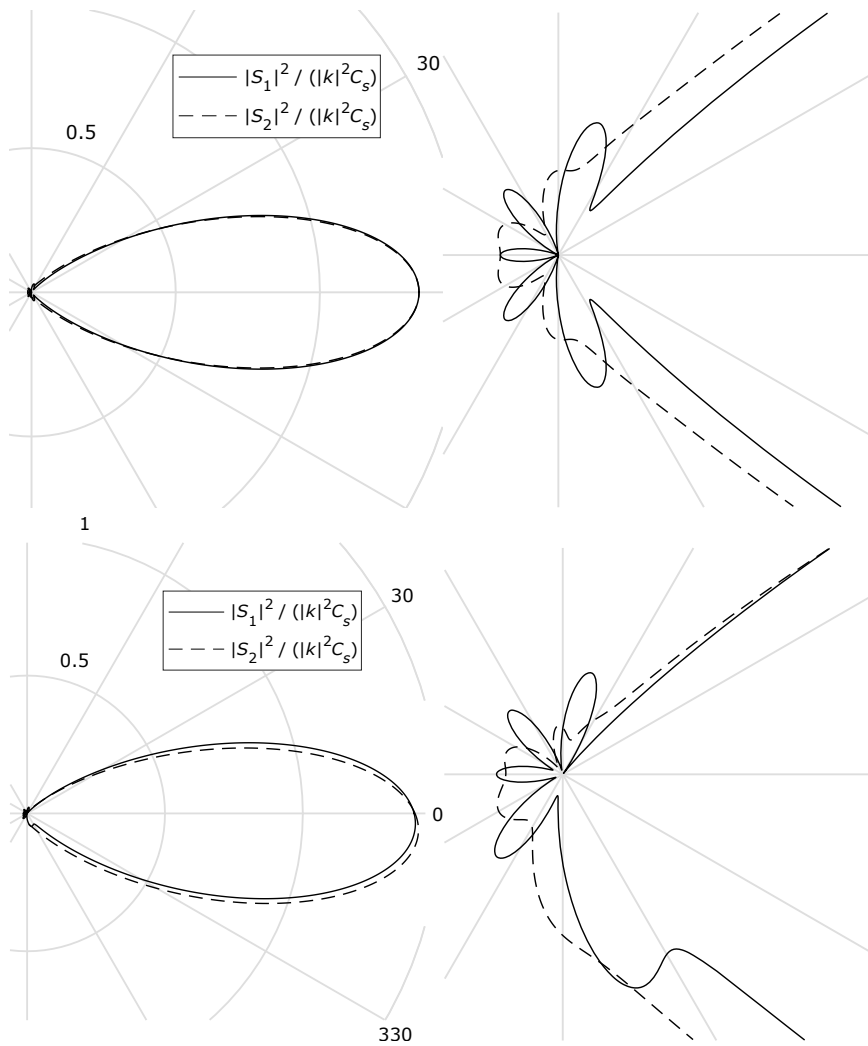

Fig. 5. The two polarization components of the phase function for a spherical particle with $x \approx 4$. The first row is the result for a homogeneous incident wave (Mie scattering). The second row is the result for an inhomogeneous wave with $\alpha \approx i 0.1$. The plots in the right column are closeups of the region around the origin of the plots in the left column.

write down new expressions for the functions $\pi_{n}$ and $\tau_{n}$ that are used in the standard expressions for the scattering components (39-40). For homogeneous and inhomogeneous plane waves, we have

$$
\begin{aligned}
\pi_{n}(\hat{\theta}) & =-\frac{\partial W_{2 n}\left(\theta, \frac{\pi}{2}\right)}{\partial \beta} \\
& =\sum_{m=0}^{n-1}(m-n) R_{n-1}^{m} P_{n}^{m+1}(0) \cos (m \hat{\theta}) \\
\tau_{n}(\hat{\theta}) & =\frac{\partial W_{1 n}\left(\theta, \frac{\pi}{2}\right)}{\partial \theta} \\
& =\sum_{m=1}^{n} m^{2} R_{n}^{m} P_{n}^{m}(0) \cos (m \hat{\theta}),
\end{aligned}
$$

where $\hat{\theta}=\theta-\hat{\alpha}$ is allowed to be a complex number and the scattering components are given by the usual equations (39-40).

\section{EVALUATION}

To provide some verification of the new, generalized equations for the angular dependency of the scattering components (5253), we first see if they reproduce results for homogeneous waves calculated using the standard equations (41-42). I do this using the same configuration as Belokopytov and Vasil'ev [18], namely $r=0.4 \mu \mathrm{m}, n_{\text {med }}=1, n_{p}=1.47+i 10^{-6}$, and $\lambda=632.8 \mathrm{~nm}$. Using 1000 equally distributed angular samples (and $\hat{\alpha}=0$ ), 


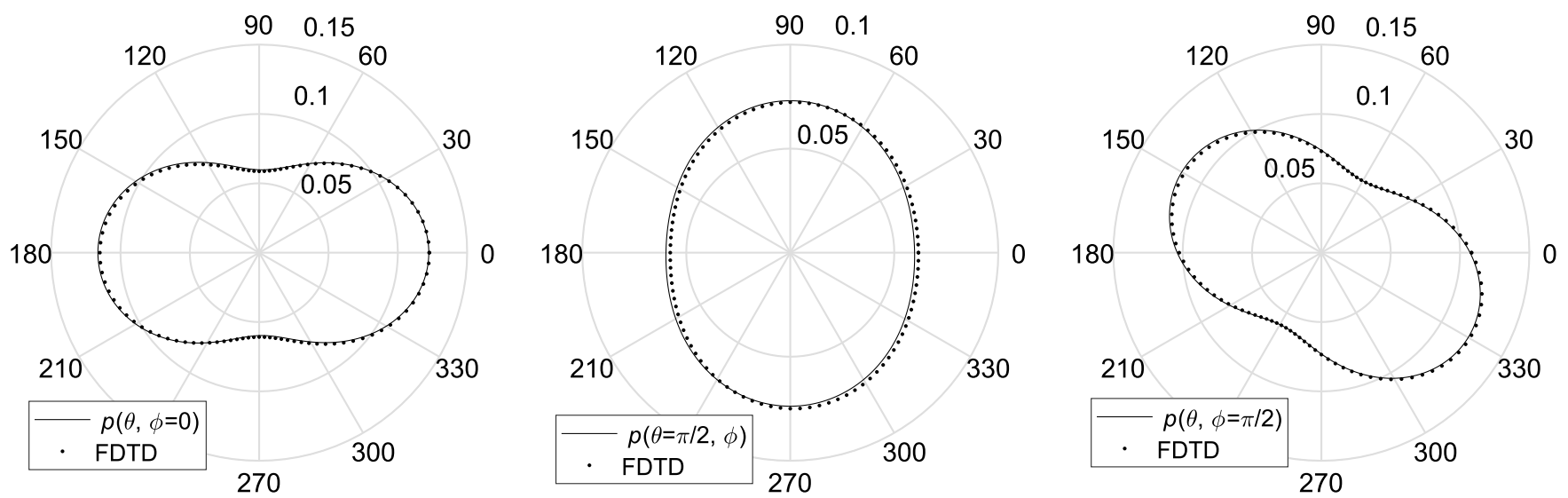

Fig. 6. Finite-difference time-domain (FDTD) simulation of the scattering by a gold particle in air of a plane wave of unpolarized light. Hypothetical wave inhomogeneity is $\hat{\alpha}=0.5$. Three planar slices of the simulated result are compared with the phase function $p(\theta, \phi)$ : (left) $\phi=0$ and varying $\theta$, (middle) $\theta=\pi / 2$ and varying $\phi$, (right) $\phi=\pi / 2$ and varying $\theta$.

the root-mean-squared difference between $\left(S_{1}, S_{2}\right)$ calculated using the standard equations (41-42) and the new equations (5253 ) was $2 \cdot 10^{-15}$, which is only one order of magnitude larger than the machine epsilon for double precision numbers. This is quite convincing given that we are working with expansions that require quite a few operations per function evaluation. The resulting scattering components are in the first row of Fig. 5.

The scattering of a homogeneous wave by a spherical particle is invariant to rotation around the forward direction. In the case of an inhomogeneous wave with wave vector $\mathbf{k}_{i}=\mathbf{k}_{i}^{\prime}+i \mathbf{k}_{i}^{\prime \prime}$, the incident wave is determined by projecting $\mathbf{k}_{i}^{\prime}$ and $\mathbf{k}_{i}^{\prime \prime}$ to the scattering plane (Eq. 45). If we produce an inhomogeneous wave by means of total internal reflection (an evanescent wave), it will exist along the surface with $\mathbf{k}_{i}^{\prime}$ tangent to the surface and $\mathbf{k}_{i}^{\prime \prime}$ normal to the surface. In the tangent plane, the projected $\mathbf{k}^{\prime \prime}$ is the zero vector, and the phase function will be the same as for the homogeneous wave (first row of Fig. 5). In the plane perpendicular to the surface, we would have $k_{z}$ real and $k_{y}$ imaginary. The second row of Fig. 5 shows the result for $k_{y}=i 1 \mu m^{-1}$, which by insertion in Eq. (19) gives $\hat{\alpha} \approx i 0.1$. The results in Fig. 5 (right column) are the same as in Fig. 2a of Belokopytov and Vasil'ev [18]. In the tangent plane, these authors find that the result for the inhomogeneous wave differs slightly from the result for the homogeneous wave. This deviation could be due to the fact that they are plotting wave intensities rather than the polarization components of the phase function.

To consolidate the idea of projection to the scattering plane, I compare with a numerical finite-difference time-domain (FDTD) simulation of a plane wave being scattered by a gold particle in air $\left(n_{\text {med }}=1\right)$. The simulation tool is FDTD Solutions by Lumerical Inc. I use two sources to model unpolarized incident light. The wave is configured to have $\lambda=525 \mathrm{~nm}$ and $\hat{\alpha}=0.5$, while the gold particle has radius $r=0.05 \mu \mathrm{m}$ and refractive index $n_{p}=0.6181+i 2.144$ [50]. The far-field scattered electric vector output from the numerical simulation was normalized so that it corresponded to a phase function. Results are in Fig. 6. The curves match each other well despite a very slight unexpected shift in the simulation (particularly noticeable in the slice that varies the $\phi$ angle). This comparison specifically tests the insight that we can use projection instead of varying the $\beta$ angle in the solution. In this hypothetical case where $\hat{\alpha}$ is real-valued, the same result is obtained if the projection method is used together with the standard Lorenz-Mie equations (41-42).

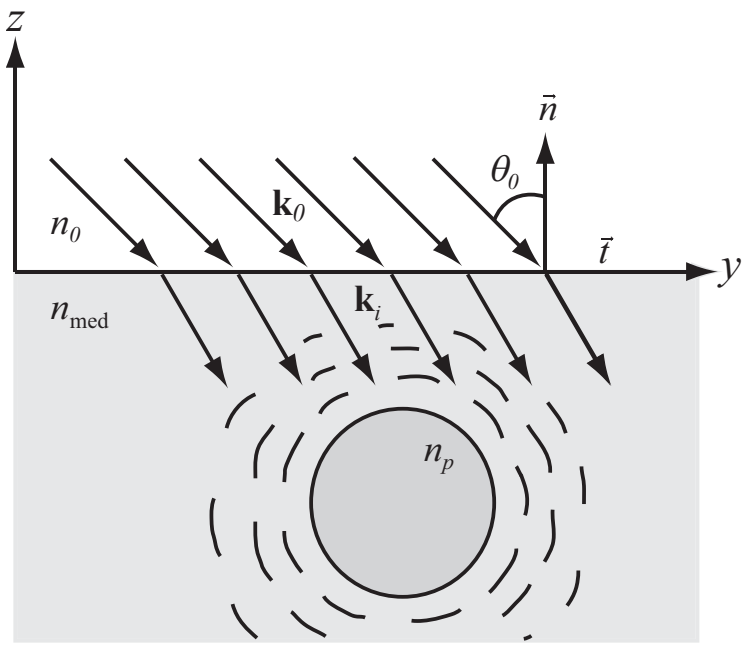

Fig. 7. Plane wave refracting into an absorbing medium at an oblique angle. The transmitted wave is then inhomogeneous, which influences how it is scattered by a particle.

The transmitted wave due to a homogeneous plane wave incident on an absorbing medium has its wave vector $\mathbf{k}_{i}$ in the plane of incidence. Even more specifically, the imaginary part $\mathbf{k}_{i}^{\prime \prime}$ is in the direction of the inward surface normal $[17,26]$. In fact, we can find the wave vector of the transmitted wave using the generalized law of refraction [26, 27]:

$$
\mathbf{k}_{i}=k_{0}\left(\vec{t} n_{0} \sin \theta_{0}-\vec{n} \sqrt{n_{\text {med }}^{2}-n_{0}^{2} \sin ^{2} \theta_{0}}\right),
$$

where $\vec{n}$ is the surface normal and $\vec{t}$ is the surface tangent in the plane of incidence. The transmitted wave is most easily described by resolving it into a TE and a TM mode. The forward directions for these modes are given by the directions of the corresponding time-averaged Poynting vectors. These are $\mathbf{k}_{i}^{\prime}$ for the TE mode and $\operatorname{Re}\left(\mathbf{k}_{i} / n_{\text {med }}^{2}\right)$ for the TM mode [26].

We can now construct an example of a plane wave refracting into an absorbing medium where it becomes inhomogeneous and is scattered by a particle (Fig. 7). Let us consider light of wavelength $\lambda=530 \mathrm{~nm}$ incident on a methanolic $0.4 \mathrm{~mol} / 1$ rhodamine $6 \mathrm{G}$ solution with $n_{\text {med }}=1.4+i 0.27$ [51]. Suppose this dye solution contains a $400 \mathrm{~nm}$ titanium dioxide particle of 
refractive index $n_{p}=2.5+i 1.4 \cdot 10^{-7}$ [52]. We then have a case that seems relevant in dye based random lasing [29-32]. The homogeneous wave in air $\left(n_{0}=1\right)$ is incident on the surface of the dye solution at a $45^{\circ}$ angle, thus $\theta_{0}=\pi / 4$. We choose a coordinate system with the surface normal in the $z$-direction and the $y z$-plane as the plane of incidence, such that $\vec{t}$ is in the $y$-direction. This is illustrated in Fig. 7, and we now have enough information to calculate $\mathbf{k}_{i}$, which we can insert in Eq. 45 .

Let us illustrate the distribution of the scattered light using two special cases: (a) the scattering plane perpendicular to the plane of incidence but containing the forward direction; and (b) the scattering plane parallel to the plane of incidence. In the first case (a), the real and imaginary parts of the projected wave vector are both parallel with the forward direction. Thus the scattering components are the same as for a homogeneous wave of light $(\hat{\alpha}=0)$. The only difference in this case is in the slightly different forward directions for the TE and TM components. In the second case (b), we have $\mathbf{k}=\mathbf{k}_{i}$. In addition, the TE component defines $E_{\perp i}$ and the TM component defines $E_{\| i}$, so that the forward directions

$$
\mathbf{S}_{i}^{\perp}=\mathbf{k}_{i}^{\prime} \quad \text { and } \quad \mathbf{S}_{i}^{\|}=\operatorname{Re}\left(\frac{\mathbf{k}_{i}}{n_{\text {med }}^{2}}\right)
$$

relate to $S_{1}$ and $S_{2}$, respectively. These two cases (a and b) are plotted in Fig. 8, where the consequence of the wave inhomogeneity is seen both in the direction of the forward peak and in the shape of the smaller lobes. As opposed to the axial symmetry of conventional Lorenz-Mie theory, the scattering distribution here only exhibits mirror symmetry. The plane of incidence $(y z)$ is the mirror plane in this example. In general, the mirror plane is the plane spanned by the real part and the imaginary part of the wave vector.

\section{DISCUSSION AND CONCLUSION}

I have introduced a practical solution for the scattering of an inhomogeneous plane wave by a particle in an absorbing medium. The solution simplifies to the existing solution for a homogeneous wave if inhomogeneity $(\hat{\alpha})$ is zero. It also simplifies to the existing solution for an inhomogeneous wave in a nonabsorbing medium if host medium absorption $\left(n_{\text {med }}^{\prime \prime}\right)$ is zero. The new equations for the angular dependency of the scattering components (Equations 52-53) are easy to evaluate numerically using recurrence relations for the associated Legendre polynomials with argument zero (Equations 47-51). This solution seems a practical alternative to the numerical techniques for solving Maxwell's equations [36] and the more involved analytical solution $[21,22]$ that one would otherwise employ.

As is natural in a phase function context, my solution employs a far field approximation. This is done in two places: the assumption of a spherical wave (11) and the approximation of the distance-dependent Riccati-Bessel function $\zeta_{n}$ and its derivative (34). It is however worth noting that the far field assumption is not required for using the key insight that makes the solution practical. The key insight is that we can expand the incident wave in a way so that we only need to evaluate associated Legendre polynomials with argument zero (rather than complex valued argument). This practical expansion should also be applicable in a vector spherical harmonics context [22], where we would presumably get computationally attractive formulae for the electromagnetic field vectors without a far field approximation (but not expressions for the scattering components as

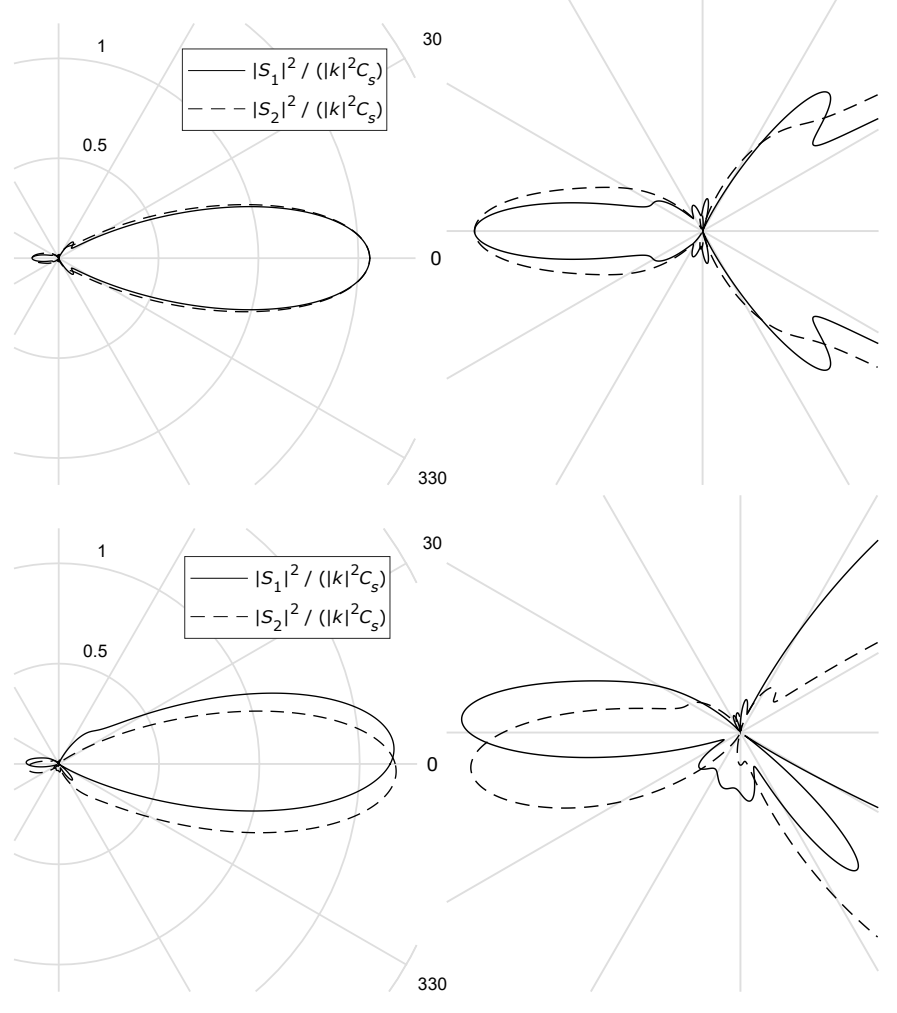

Fig. 8. Polarization components of the phase function for a spherical titanium dioxide particle submerged in a laser dye medium (as illustrated in Fig. 7). The rows contain the two cases ( $a$ and $b$ ) described in the main text. With the standard theory for homogeneous waves (Mie scattering), the result in the second row (b) would be the same as in the first row with swapped $S_{1}$ and $S_{2}$. The plots in the right column are closeups of the region around the origin of the plots in the left column.

presented here). I leave an investigation of a vector spherical harmonics solution based on this expansion for future work.

The provided phase function solution seems useful as plane waves are most often inhomogeneous when propagating in an absorbing medium. A radiative transfer simulation should account for the influence of this inhomogeneity on the directions of the scattered light. This is particularly relevant because the scattering of light by particles in absorbing media has recently found interesting applications in random lasing and solar cell absorption enhancement. I provided an example to illustrate how scattering is affected by wave inhomogeneity in a configuration similar to those seen in dye based random lasing.

\section{REFERENCES}

1. L. Lorenz, "Lysbevægelser i og uden for en af plane Lysbølger belyst Kugle," Det kongelige danske Videnskabernes Selskabs Skrifter 6, 1-62 (1890). 6. Række, naturvidenskabelig og mathematisk Afdeling VI. 1.

2. G. Mie, "Beiträge zur Optik trüber Medien, speziell kolloidaler Metallösungen," Annalen der Physik 25, 377-445 (1908). IV. Folge.

3. M. Kerker, The Scattering of Light and Other Electromagnetic Radiation (Academic Press, New York, 1969).

4. W. C. Mundy, J. A. Roux, and A. M. Smith, "Mie scattering by spheres in an absorbing medium," Journal of the Optical Society of America 64, 1593-1597 (1974).

5. P. Chýlek, "Light scattering by small particles in an absorbing medium," Journal of the Optical Society of America 67, 561-563 (1977). 
6. C. F. Bohren and D. P. Gilra, "Extinction by a spherical particle in an absorbing medium," Journal of Colloid and Interface Science 72, 215221 (1979).

7. M. Quinten and J. Rostalski, "Lorenz-Mie theory for spheres immersed in an absorbing host medium," Particle \& Particle Systems Characterization 13, 89-96 (1996).

8. A. N. Lebedev, M. Gartz, U. Kreibig, and O. Stenzel, "Optical extinction by spherical particles in an absorbing medium: Application to composite absorbing films," The European Physical Journal D - Atomic, Molecular and Optical Physics 6, 365-373 (1999).

9. Q. Fu and W. Sun, "Mie theory for light scattering by a spherical particle in an absorbing medium," Applied Optics 40, 1354-1361 (2001).

10. I. W. Sudiarta and P. Chylek, "Mie-scattering formalism for spherical particles embedded in an absorbing medium," Journal of the Optical Society of America A 18, 1275-1278 (2001).

11. P. Yang, B.-C. Gao, W. J. Wiscombe, M. I. Mischenko, S. E. Platnick, H.-L. Huang, B. A. Baum, Y. X. Hu, D. M. Winker, S.-C. Tsay, and S. K. Park, "Inherent and apparent scattering properties of coated or uncoated spheres embedded in an absorbing host medium," Applied Optics 41, 2740-2758 (2002)

12. G. Videen and W. Sun, "Yet another look at light scattering from particles in absorbing media," Applied Optics 42, 6724-6727 (2003).

13. Q. Fu and W. Sun, "Apparent optical properties of spherical particles in absorbing medium," Journal of Quantitative Spectroscopy and Radiative Transfer 100, 137-142 (2006).

14. J. Randrianalisoa, D. Baillis, and L. Pilon, "Modeling radiation characteristics of semitransparent media containing bubbles or particles," Journal of the Optical Society of America A 23, 1645-1656 (2006).

15. J. Yin and L. Pilon, "Efficiency factors and radiation characteristics of spherical scatterers in an absorbing medium," Journal of the Optical Society of America A 23, 2784-2796 (2006).

16. T. C. Fry, "Plane waves of light I. Electromagnetic behavior," Journal of the Optical Society of America 15, 137-161 (1927).

17. T. C. Fry, "Plane waves of light II. Reflection and refraction," Journal of the Optical Society of America 16, 1-25 (1928).

18. G. V. Belokopytov and E. N. Vasil'ev, "Scattering of a plane inhomogeneous electromagnetic wave by a spherical particle," Radiophysics and Quantum Electronics 49, 65-73 (2006).

19. D. C. Prieve and J. Y. Walz, "Scattering of an evanescent surface wave by a microscopic dielectric sphere," Applied Optics 32, 1629-1641 (1993).

20. J.-M. Jin, The Finite Element Method in Electromagnetics (Wiley, 2014), 3rd ed.

21. F. Frezza and F. Mangini, "Electromagnetic scattering by a buried sphere in a lossy medium of an inhomogeneous plane wave at arbitrary incidence: spectral-domain method," Journal of the Optical Society of America A 33, 947-953 (2016).

22. F. Frezza and F. Mangini, "Vectorial spherical-harmonics representation of an inhomogeneous elliptically polarized plane wave," Journal of the Optical Society of America A 32, 1379-1383 (2015).

23. W. H. Press, S. A. Teukolsky, W. T. Vetterling, and B. P. Flannery, Numerical Recipes in C: The Art of Scientific Computing (Cambridge University Press, Cambridge, 1992), 2nd ed.

24. J. A. Doornik, "Numerical evaluation of the Gauss hypergeometric function by power summations," Mathematics of Computation 84, 18131833 (2015).

25. T. C. Fry, "Plane waves of light III. Absorption by metals," Journal of the Optical Society of America 22, 307-332 (1932).

26. E. E. Bell, "Optical constants and their measurement," in "Light and Matter la,", vol. XXV/2a of Handbuch der Physik (Encyclopedia of Physics) S. Flügge and L. Genzel, eds. (Springer-Verlag, Berlin, 1967), chap. 1, pp. 1-58.

27. M. A. Dupertuis, M. Proctor, and B. Acklin, "Generalization of complex Snell-Descartes and Fresnel laws," Journal of the Optical Society of America A 11, 1159-1166 (1994).

28. G. Gouesbet and G. Gréhan, Generalized Lorenz-Mie Theories (Springer, 2017), 2nd ed.

29. N. M. Lawandy, R. M. Balachandran, A. S. L. Gomes, and E. Sauvain,
"Laser action in strongly scattering media," Nature 368, 436-438 (1994).

30. X. Wu, W. Fang, A. Yamilov, A. Chabanov, A. Asatryan, L. Botten, and $\mathrm{H}$. Cao, "Random lasing in weakly scattering systems," Physical Review A 74, 053812-1-053812-11 (2006).

31. J. Yi, G. Feng, L. Yang, K. Yao, C. Yang, Y. Song, and S. Zhou, "Behaviors of the Rh6G random laser comprising solvents and scatterers with different refractive indices," Optics Communications 285, 5276-5282 (2012).

32. F. Luan, B. Gu, A. S. L. Gomes, K.-T. Yong, S. Wen, and P. N. Prasad, "Lasing in nanocomposite random media," Nano Today 10, 168-192 (2015).

33. J.-Y. Lee and P. Peumans, "The origin of enhanced optical absorption in solar cells with metal nanoparticles embedded in the active layer," Optics Express 18, 10078-10087 (2010).

34. J. R. Nagel and M. A. Scarpulla, "Enhanced absorption in optically thin solar cells by scattering from embedded dielectric nanoparticles," Optics Express 18, A139-A146 (2010).

35. I. Kim, K.-S. Lee, T.-S. Lee, D. S. Jung, W.-S. Lee, W. M. Kim, and K.-S Lee, "Size dependence of spherical metal nanoparticles on absorption enhancements of plasmonic organic solar cells," Synthetic Metals 199 174-178 (2015).

36. S. Yadollahzadeh, S. Alavizadeh, and H. Baghban, "Absorption enhancement in thin-film photoluminescence layers with metal nanoparticles inter-coupling engineering," Optics and Spectroscopy 118, 930-935 (2015).

37. R. K. Harrison and A. Ben-Yakar, "Point-by-point near-field optical energy deposition around plasmonic nanospheres in absorbing media," Journal of the Optical Society of America A 32, 1523-1535 (2015).

38. J. C. Maxwell, $A$ Treatise on Electricity and Magnetism (Clarendon Press, 1873). Two volumes.

39. C. F. Bohren and D. R. Huffman, Absorption and Scattering of Light by Small Particles (John Wiley \& Sons, Inc., 1983).

40. J. H. Poynting, "On the transfer of energy in the electromagnetic field," Philosophical Transactions of the Royal Society of London 175, 343-361 (1884).

41. M. Born and E. Wolf, Principles of Optics: Electromagnetic Theory of Propagation, Interference and Diffraction of Light (Cambridge University Press, 1999), seventh (expanded) ed.

42. H. C. van de Hulst, Light Scattering by Small Particles (John Wiley \& Sons, Inc., New York, 1957).

43. G. Bauer, "Von den Coefficienten der Reihen von Kugelfunctionen einer Variablen," Journal für die reinen und angewandte Mathematik 56 $101-121$ (1826)

44. M. Abramowitz and I. A. Stegun, eds., Handbook of Mathematical Functions With Formulas, Graphs, and Mathematical Tables, vol. 55 of Applied Mathematics Series (National Bureau of Standards, Washington, D.C., 1964). Tenth printing, December 1972, with corrections.

45. P. Debye, "Der Lichtdruck auf Kugeln von beliebigem Material," Annalen der Physik 335, 57-136 (1909).

46. J. V. Dave, "Scattering of electromagnetic radiation by a large, absorbing sphere," IBM Journal of Research and Development 13, 302-313 (1969).

47. G. W. Kattawar and G. N. Plass, "Electromagnetic scattering from absorbing spheres," Applied Optics 6, 1377-1382 (1967).

48. W. J. Wiscombe, "Improved Mie scattering algorithms," Applied Optics 19, 1505-1509 (1980).

49. J. R. Frisvad, N. J. Christensen, and H. W. Jensen, "Computing the scattering properties of participating media using Lorenz-Mie theory," ACM Transactions on Graphics 26 (2007). Article 60.

50. P. B. Johnson and R. W. Christy, "Optical constants of the noble metals," Physical Review B 6, 4370-4379 (1972).

51. Y. Lu and A. Penzkofer, "Absorption behaviour of methanolic rhodamine $6 \mathrm{G}$ solutions at high concentrations," Chemical Physics 107, 175-184 (1986).

52. T. Siefke, S. Kroker, K. Pfeiffer, O. Puffky, K. Dietrich, D. Franta, I. Ohlídal, A. Szeghalmi, E.-B. Kley, and A. Tünnermann, "Materials pushing the application limits of wire grid polarizers further into the deep ultraviolet spectral range," Advanced Optical Materials 4, 1780-1786 (2016). 